\title{
Performance Evaluation of Throughput and Fairness Balancing in LTE Packet Scheduling
}

\author{
Alaa Omer Najim ${ }^{1}$, Nor Asilah Wati Abdul Hamid ${ }^{1}$, Idawaty Ahmad ${ }^{1}$, Zurina Mohd \\ Hanapi $^{1}$ \\ alaaomer2006@yahoo.co.uk ,\{asila, idawaty, zurinamh@upm.edu.my $\}$
}

\author{
Department of Communication Technology and Network, Faculty of computer Science and \\ Information Technology, Universiti Putra Malaysia, 43400 UPM, Serdang, Selangor D.E., Malaysia ${ }^{1}$
}

\begin{abstract}
Provisioning network QoS is the essential aim in the recent cellular broadband system e.g. 4G LTE network. However, this task became a conflict when variety of user application introduced to the network, having that; each of which should be maintained with different QoS requirements. In another side, when radio resources are limited, that is very often in mobile networks nowadays, maximizing system capacity will be obtained on expense of unfair share of the resources. Therefore, motivated by the highlighted issue above, we emphasize on balancing between throughput and fairness as a key design criterion in LTE scheduling scheme. We also handle a discussion for some of the common scheduling schemes based on the above goal. A system level simulation is set up to evaluate which of the investigated schedulers can maintain the bond of fairnessthroughput with a good performance.
\end{abstract}

Keywords: LTE, Downlink scheduling, QoS, Scheduling algorithm, FairnessThroughput trade-off, simulation.

\section{Introduction}

Long Term Evolution (LTE) mobile broadband networks have gained extensive attentions in research and industry. LTE is basically introduced by the 3GP P in order to satisfy the growing demands of data transmission [1]. As a step to Fourth Generation of mobile technology, LTE is capable of providing a high user expe rience of delivering multimedia and Internet applications even in high-mobility scenarios. Therefore, common network performance indices such as throughput, low delay, and complicated traffic models are most likely considered compared to the previous cellular networks. LTE implements Orthogonal Frequency Division Multiple Access (OFDMA) as an access technology for the downlink channel, as well as developed base station named evolved-NodeB (eNB) that delivers high data rate with lower latency values for different users who are multiplexed in time and frequency domains [2]. Thus, resources scheduling procedure at eNB should be given a due care to achieve the required performance and a high satisfaction level of the end users.

The issue of packet scheduling has been existed over all the generations of wireless and mobile networks. As more multimedia from Real Time (RT), and Non-Real Time (NRT) traffic applications are supplied by the base station, Quality of Service (QoS) for these application became diverse and with specific characteristics that needs to be fulfilled. In LTE 
protocols stack, MAC layer is the place where all the scheduling procedures and decisions are made. Whereby, these decision became critical when prioritizing mixed user flows exist in a single network traffic scenario. Technically, different user applications have different characteristics, and thus; need different specifications of QoS features to be satisfied. For RT traffic, low delay and data loss while maintaining a good throughput are the main QoS metrics that should be guaranteed [1]. Ironically, NRT applications can compromise more delay for the favor of a moderate to high data rate. Meanwhile, fairness and system throughput trade-off should be maintained between RT and NRT to provide a realistic scheduler design. This is turn brings a challenge for the developing scheduling policies for providing QoS awareness when handling mixed traffic.

In this paper, with respect to realize the aim of enhancing the network utilization and the capacity in scenarios of different user flow with diverse QoS, we comprehensively investigate some of the well-known scheduling policies in for LTE Downlink (DL) Frequency Domain (FD) scheduling. As a part of the paper goal we emphasize on how efficient is the scheduling policy in maintaining the trade-off between fair resources share and boosting higher throughput. The significance of this work lies in providing an informative analysis that contributes to the design of an effective packet scheduling technique for the downlink channel of LTE network that supports both RT and NRT traffic. The remainder of this paper is organized as follows. Section II describes the downlink system scheduling model in LTE, followed by discussions on throughput-fairness trade-off in common scheduling schemes handled in Section III. The simulation experiment scenario is discussed in Section IV, while Section V discusses the obtained results of the scheduling algorithms, and finally, section VI concludes the paper.

\section{Downlink System Scheduling in LTE}

Among the major reasons why LTE is a $4 \mathrm{G}$ and beyond mobile platform is that in LTE user channel, resources allocation procedures are possible in both Time Domain (TD) and FD. This indeed provides a variety of QoS dimensions to be improved when designing a scheduling algorithm [3]. Basically, the process of resources scheduling at the MAC layer is iterated at 3 Time Transmission Interval (TTI) time, the scheduler should know the channel gain for each sub-carrier and each user. However, due to limited signaling channel resources, sub-carriers are grouped into RBs, each consisting of 12 adjacent sub-carriers as shown in Figure 1. [4]. Each RB has a time slot duration of $0.5 \mathrm{~ms}$, which corresponds to 6 or 7 OFDM symbols depending on whether an extended or normal cyclic prefix is used. The smallest resource unit that a scheduler can allocate to a user is physical resource block (PRB), which consists of two consecutive Resource Blocks (RBs), spanning a sub-frame time duration or Transmission Time Interval (TTI) of $1 \mathrm{~ms}$ and a bandwidth of $180 \mathrm{kHz}$. 


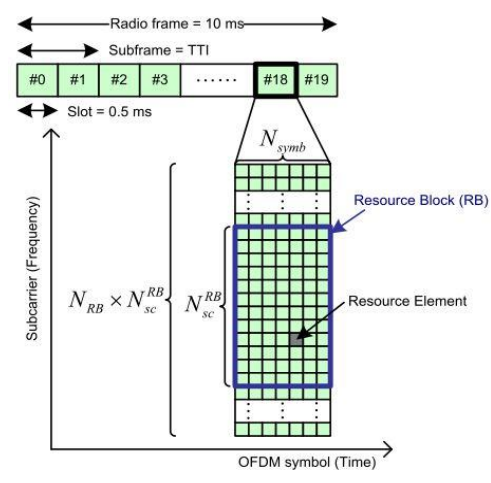

Fig. 1. LTE frame structure in TD/FD.

The process of DL packet scheduling in LTE network is a sequence of procedures that are repeatedly triggered every TTI, and involves both UE and eNB.It is initiated when UE measures its Channel Quality Indicator (CQI) and sends it to eNB via UL channel as depicted in Figure 2. [5]. CQI and QoS parameters from the RLC and Application layers are then used by eNB in allocation decisions and distributing RBs among flows. A part of that, selecting the suitable Modulation and Coding Scheme (MCS) in the physical layer provides high performance with lower block errors. Finally, results of scheduling decisions and the session information above are sent back to UE through Packet Data Control Channel (PDCCH), who engages in proper Packet Data Shared Channel (PDSCH) payload if it is scheduled.

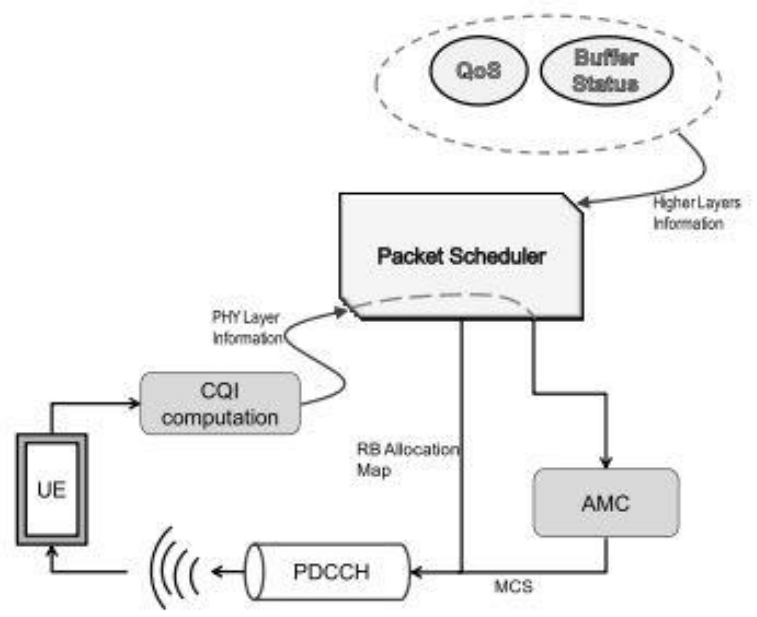

Fig. 2. Basic model of downlink packet scheduling process in LTE. 


\section{Throughput-Fairness trade-off in the common Scheduling Schemes}

Provisioning QoS of LTE network of various traffic types is an essential goal when deploying eNB model. This can be realized by assuming tight QoS criteria in the packet scheduler model that is designed for the eNB. When operating on scarce of bandwidth resources, this goal became a challenge to be achieved. Whereby, increasing the system throughput while maintain a good level of fairness is an issue which results in a normal tradeoff between the two metrics. Therefore, we emphasize in this article the importance of building this bond as a key design aspect for developing scheduling model in LTE.

Literature surveys and reviews are very limited in clearly investigating the aspect of relationship between throughput and fairness [6][7][8], meanwhile majority of [9][10][11][12][13] discuss the overall QoS performance of well-known scheduling schemes. This in turn drive many LTE scheduling methods proposals from the literature not to be aware of maintaining a balance between throughput and fairness in the design phases, which can be obviously noticed when operating scenarios of high load traffic. For example in [14][15][16] if variable traffic volume is assumed, this balance is gradually deteriorated. In addition, some studies [17] reveals a humble fairness level; however this exposes an issue of complexity that affects the possibility of have a real-implementation scenario. In [18], the new proposed scheduler is more complex than the Proportional Fair algorithm in order to increase the overall system throughput respecting fairness approach. Other scheme can provide satisfactory scheduling performance for multimedia services [19]. In [20], scheme is suitable for real-time traffic, especially for the video traffic transmissions. The proposed allocation scheme that has better performance with respect to packet loss rates and delay has been concluded, achieved by exploiting the deadline properties [21]. In the following context, we investigate some of common scheduling algorithms based on throughput/fairness balancing level.

\subsection{Proportional Fairness (PF) Scheduler}

One of the classical fairness standards maintains a trade-off between maximum rate and fairness that was initially proposed in [22]. The fairness concept in PF is derived from Round Robin fairness principle. Thus it attempts to schedule Ues with good CQIs, meanwhile preventing other users from starving by imposing a fairness level of rate distributions. The scheduler assigns RBs to flows with highest priority. This priority is obtained from the following metric, see equation (1)

$$
\begin{gathered}
x_{i, n}^{P F}=\frac{d_{n}^{i}(t)}{\overline{\mathrm{R}}(\mathrm{t}-1)} \\
\overline{\mathrm{R}}_{i}(t-1)=(1-\beta) r^{i}(\mathrm{t})+\beta \overline{\mathrm{R}}_{i}(\mathrm{t}-1) \\
T^{\mathrm{f}}=\frac{1}{1+\beta}
\end{gathered}
$$

Where, $d_{n}^{i}(t)$ is the estimated data rate for the $\mathrm{i}^{\text {th }}$ flow at time $\mathrm{t}$ on the $\mathrm{n}^{\text {th }} \mathrm{RB}$, and $\mathrm{R}_{\mathrm{i}}(\mathrm{t})$ is the previous average data rate if the $\mathrm{i}^{\text {th }}$ user flow at time $t$. From the above equations we observe that $\mathrm{PF}$ mechanism depends on previous average throughput which acts as a 
weighting factor of the incoming data rate. Hence, users with low CQIs still can be served within amount of time. The parameter $\beta$ in equation (2) plays an important role, as it is impacts the time window $\mathrm{T}^{\mathrm{f}}$ value in equation (3) where fairness should be imposed according to the relation.

\subsection{EXPonential-PF (EXP-PF) Scheduler}

In scenarios where delay sensitive traffic are coexisted, PF starts treating all flows with the same manner which ends up in deteriorating performance of RT traffic. Based on that, authors in [23] modified PF principle to have a flexible behavior with RT/NRT traffic. EXP/PF is integration between PF and a developed EXP term. EXP properties guarantee the throughput for RT services and PF properties maintaining a minimum level of service for NRT to keep balanced service fairness. EXP/PF metric tightly depends on the service type that prioritized by the RT metric,

$$
x_{i, n}^{E X P / P F}=\exp \left(\frac{\alpha_{i} q_{i}-w}{1+\sqrt{w}}\right) \frac{d_{n}^{i}(t)}{\overline{\mathrm{R}}_{i}(t-1)}
$$

Where and $\alpha_{\mathrm{i}}$ are given from

$$
\begin{gathered}
w=\frac{1}{N_{R T}} \sum_{i=1}^{N_{R T}} \alpha_{i} q_{i} \\
\alpha_{i}=\frac{\log \delta_{i}}{\tau_{i}}
\end{gathered}
$$

$\mathrm{q}_{\mathrm{i}}$ is the queue size for $\mathrm{i}^{\text {th }}$ flow is the weight factor for the exponential termthat enables maximizing throughput with fairness maintenance, as it counts for the average values of $q_{i} . \alpha_{i}$ is a system parameter obtained from the acceptable packet loss rate for the $\mathrm{i}^{\text {th }}$ user, $\delta_{\mathrm{i}}$, over the maximum delay bound $\tau_{\mathrm{i}}, \alpha_{\mathrm{i}}$ here weights the flows with the higher requirements in terms of accepted data rate. The metric for NRT is the typical PF rule, see equation (4), (5), (6). From the above discussion, it is noticed that EXP/PF attempts to give due care to RT flows by tightly considering fairness throughout imposing PF term and recording average values of queue sizes, Meanwhile targeting high throughputs by prioritizing flows with the large queue size. For NRT fairness and minimum data rate service can be achieved by applying PF.

\subsection{EXPonential-Rule (EXP-Rule) Scheduler}

When firstly introduced EXP-Rule [24] is defined as a throughput-optimalscheduling scheme for High Data Rates (HDR) networks. Thus, thereafter, it attracted a lot of interest by utilizing it in developing more complicated scheduling decisions. EXP-Rule is considered an enhancement of EXP/PF scheme that it highly prioritizes flows based on their achieved throughput. EXP-Rule is a service based priority metric, whereby, for RT it uses, see equation (7)

$$
x_{i, j}^{E X P / R u l e}=b_{i} \exp \left(\frac{\alpha_{\mathrm{i}} \mathrm{q}_{\mathrm{i}}}{\mathrm{c}+\sqrt{\left(1 / N_{R T}\right) \Sigma_{\mathrm{j}} \mathrm{q}_{\mathrm{j}}}}\right) \varphi^{i}
$$


Where, $\phi$ is the spectral efficiency of $i^{\text {th }}$ UE on $n^{\text {th }}$ RB. $a, b$, and $c$ are called the optimality parameters, that according to [25], set to $\left[5 /\left(0.99 \tau_{\mathrm{i}}\right), 10 /\left(0.99 \tau_{\mathrm{i}}\right)\right], 1 / \mathrm{E}\left[\phi_{\mathrm{i}}\right]$, and 1 respectively. EXP-Rule tries to equalize the weighted queue size of all the flows queues, so that if a queue is with bigger size than others, the EXP term became bigger, that in turn enables the flow to be prioritized based on the archived system throughput. On the other hand, a flow with smaller queue size than others results in small value of EXP term that is close to 1; in this case the rule behavior is toward maintaining fairness for the flow. For NRT flows, PF metric is used.

\subsection{Log-Rule Scheduler}

The Log-Rule is introduced as an opportunistic delay-based scheme by [26]. Contrasting with EXP-Rule explained above, Log-Rule prioritizes flows in way that neglecting balancing of unequal queue sizes in favor of building a trade-off between low delay amount and good level of throughput. The scheduling metric of $\log -$ Rule to prioritize RT services is, see equation (8)

$$
x_{i, n}^{\log -\text { Rule }}=b_{i} \log \left(c+\alpha_{i} q_{i}\right) \varphi_{n}^{i}
$$

Where, optimality parameters a, b, and c have the same values as in EXP - Rule.

\section{Simulation Experiment Scenario Setup}

A system level simulation experiments has been done to evaluate the performance of previously explained scheduling schemes (PF, EXP-PF, EXP-Rule, and Log-Rule). For that, an open source simulator namely, LTE-Sim [27] is utilized to run the experiments. In FD, a bandwidth of $5 \mathrm{MHz}$ is selected for the downlink channel with $25 \mathrm{RBs}$ in each TTI. In TD, resource allocation process occurs every TTI $($ TTI $=1 \mathrm{~ms})$. UEs are uniformly distributed within the eNB transmission range with distance limits between $500-1000 \mathrm{~m}$ from the eNB, and move with UE speed of $30 \mathrm{Km} / \mathrm{h}$ in a random way point mobility model as shown in Figure. 3 [28].

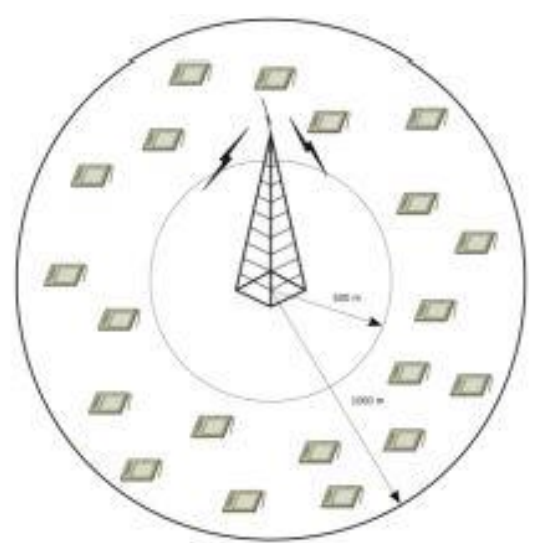

Fig. 3. System model of the simulation scenario. 
Traffic sources used are RT Video, RT VoIP and NRT flows. For Video flows, Traced Based traffic generator is used to create flows with video type of H.264 with data rate of $242 \mathrm{kbps}$. While the VoIP flows are modeled by G.729 VoIP generator, with ON OFF process where the ON period is exponentially distributed with mean of $3 \mathrm{~s}$, and the OFF period is an exponential probability density function with upper bound of $6.9 \mathrm{~s}$ mean value of $3 \mathrm{~s}$. During On period the sources sends voice traffic with a data rate of $8.4 \mathrm{kbps}$, while in the OFF period data rate is zero. Table 1 depicts the description for rest of the parameters implemented within the scenario.

\section{Numerical Results Discussion}

In this section we discuss about the simulation results of the four schedulers explained in the previous section. To the best of our knowledge, EXP-PF, EXP-Rule, and Log-Rule are implemented as throughput based scheduling policies, hence, instead of utilizing delay as QoS parameters in the metric formulation, queue size is rather placed to give a throughputorientation to the scheduler. The aim of simulation is to discuss the balance level in boosting system throughput while maintain a good fairness index with flows of each traffic type. Simulation parameters are listed in Table 1.

Table 1. Description of Simulation Parameters.

\begin{tabular}{ll}
\hline Parameters & Assumption \\
\hline Simulation duration & $100 \mathrm{~s}$ \\
Carrier frequency & $1.9 \mathrm{GHz}[27]$ \\
Bandwidth & $5 \mathrm{MHz}(25 \mathrm{RBs}$ in each TTI $)$ \\
Number of Ues & $20,40,60,80,100$ \\
UE speed & $30 \mathrm{Km} / \mathrm{h}$ \\
eNB transmission radius & $1 \mathrm{Km}$ \\
Number of cells and eNB & Single cell with $1 \mathrm{eNB}$ \\
Transmission power at eNodeB & $30 \mathrm{Km} 43 \mathrm{dBm} / \mathrm{h} \mathrm{[27]}$ \\
Path loss & $28.6+35 \log 10(1000) \mathrm{db}[27]$ \\
Thermal noise density & $174 \mathrm{dBm} / \mathrm{Hz}[27]$ \\
Noise figure & $7 \mathrm{~dB}[27]$ \\
Max Re-transmission number & $5[27]$ \\
\hline
\end{tabular}

\subsection{Fairness Index}

In this study, fairness is measured between flows of each traffic type using Jains fairness formula [29]. As presented in Figure 4. (a, b and c), fairness index with respect to the number of UEs. Log-Rule appears to be best choice for providing fairness within RT traffic applications as the logarithmic feature in Log-Rule metric provides more robustness and steadiness in considering different flow types (either heavy or light). Although EXP-PF provides a similar level of fairness as Log-Rule for RT video flows, it cannot maintain this pattern in other traffic type (RT VoIP and Best effort). In another side, EXP-Rule plots a close behavior as EXP-PF for VoIP and best effort fairness, while it is decreased for video flows. 
The exponential term used in the metric guarantees a service chance for flows with different CQIs. For EXP-PF , exponential term considers the average weight of the previous queue size for every admitted UE. Thus, it is able to provide extra fairness level when compared to EXPRule that is built to be a throughput-optimal policy.
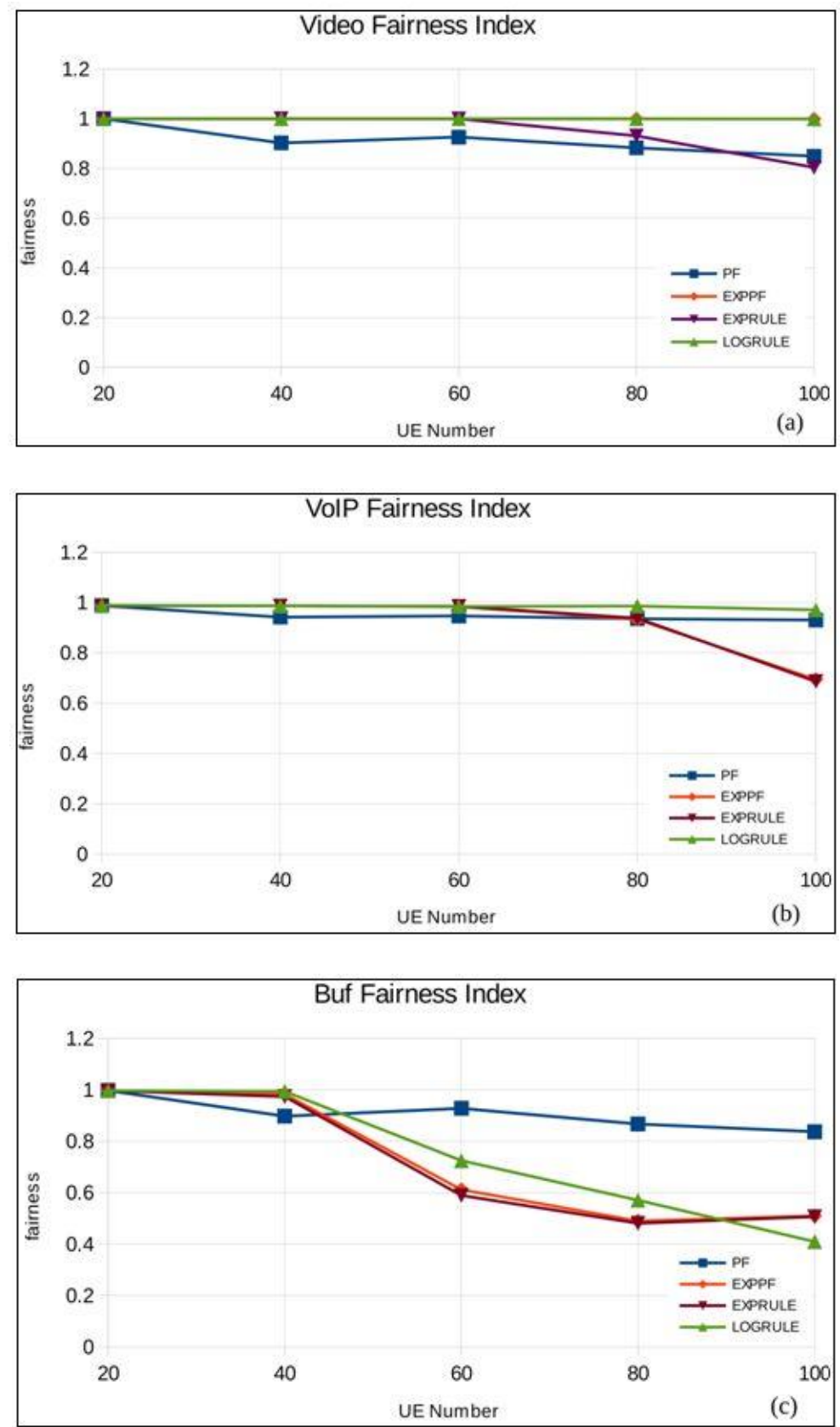

Fig. 4. Fairness Index for (a) video, (b) VoIP, and Inf. Buf traffic types 
PF on the other hand is simple fairness policy that can be noticed effective in scenarios with single traffic type. In such a multi-traffic scenario with RT traffics, its fairness behavior is decreased heavily for RT flows, while it is comparable with other scheduler. The fairness concept in PF comes from taking account of the previous data rate of the UE $\mathrm{s}$ flow. That is, if a UE flow does not has enough bandwidth to be transmitted in the previous interval, it will has a high possibility to be selected by the next interval, keeping in mind its historical data rate. This behavior obviously improves the service fairness among UEs flows under each application.

\subsection{System Throughput}

System throughput is measured as the summation of data size successfully arrived to the destination over simulation time. In this context, we describe the end-to-end delivery of packets that travel from application layer of the eNB all the way to the application layer of the respective UE. As shown in Figure. 5 ( $a, b$ and c), the aggregated flow throughput (class throughput) of different traffic types is illustrated over the increased UE sets. For RT Video streaming, packets are transmitted in bursts. Thus, a scheduler like EXP-Rule that takes account of the actual queue size in such a way that makes scheduler prioritizes flows based on high value of queue size (e.g. Video) shows the highest throughput trend. However, small RT flows (e.g VoIP) and NRT traffic has a limited chance to be transmitted. EXP-PF shows comparable results with EXP-Rule over RT traffic types. Whereby, when the cell is fully loaded, EXP-PF attempts to conserve portion of bandwidth from video to favor UE flows with low CQI Ues (that have relatively low throughputs) or other small RT flows. The difference between EXP-Rule and EXP-PF, is that when the EXP part is small and close to 1, EXP-Rule acts as Maximum Throughput scheduler, that only consider high CQI UEs, while EXP-PF act as PF. Besides that, Log-Rule is a robust and low sensitive policy that behaves with more consistency toward the variability of flows volumes. Based on that, its behavior is more conservative. For example, within video flows, the logarithmic part enables the scheduler to consider all admitted flows when assigning RB in such a way to provide relatively average throughput. This allows flows from other traffic types to be transmitted with acceptable data rates levels when comparing to exponential-based schedulers. With this advantage, small RT traffic flows like VoIP can be delivered with a good throughput. Even for NRT flows, Log-Rule allows a portion of the bandwidth to transmit these flows. For PF, the worst situation is in terms of video traffic, due to the variability nature of this traffics kind that restricts the scheduler to allocate bandwidth resources to high CQI UEs. Therefore, Ues flows are treated based on equality of sharing bandwidth. For RT VoIP, PF tends to keep a steady behavior that goes better than EXP-based scheduler as more load introduced. Meanwhile, for NRT ; since there is no variability in traffic and flows are almost equal in their sizes, $\mathrm{PF}$ is the best choice for boosting throughput. 

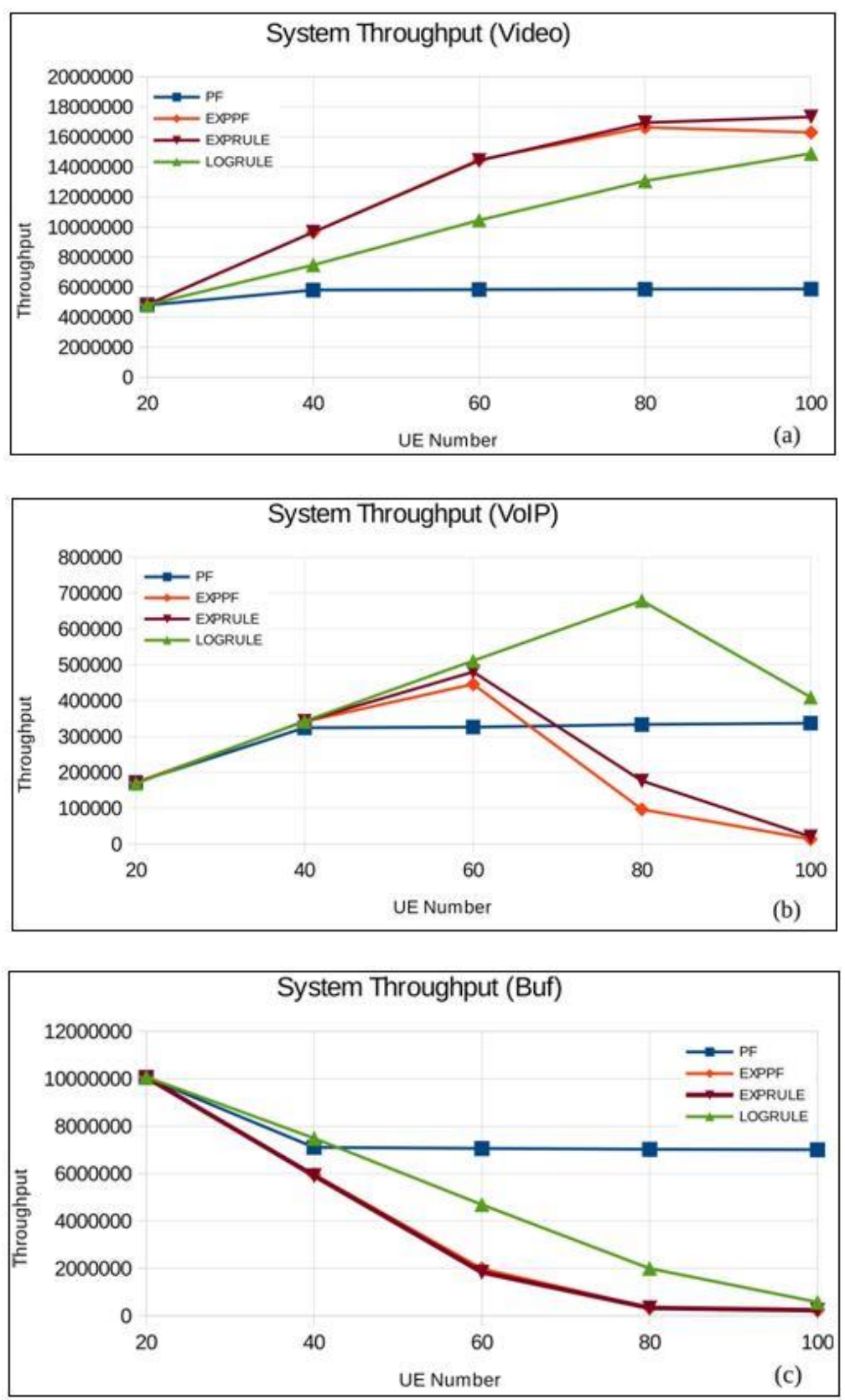

Fig. 5. System Throughput for (a) video, (b) VoIP, and Inf. Buf traffic types 


\subsection{Spectrum Efficiency}

It defines efficiency level for a scheduler to assign the available RBs to the user flows with the highest channel qualities in such a way to increase the system capacity. It is measured from the delivery of whole traffic operates through the eNB to different UEs. Thus it is a reflection of the system throughput. Figure. 6 presents the cell spectral efficiency over traffic load in eNB. Generally, EXP-PF and EXP-Rule plot comparable results, having EXP-PF performs better when the cell operates in average load, while EXP-Rule still increases the spectral efficiency in high cell load due to the feature of being throughput optimal rule. Log Rule and PF demonstrate a steady performance; that is Log-Rule schedules flows with better resources allocation decisions and slightly increases with load. PF deteriorates RBs allocation efficiency when numbers of UEs>20 and maintain a consistently the lowest behavior, as it fails to contribute in video flows throughput.

From the numerical results plotted above, we infer that the involved scheduling strategies tend to maintain a relation between measured fairness level and the obtained throughput for varied traffic types. In PF, it is obvious that the fairness behavior is directly improves as the throughput decreases. This is because the priority metric is determined as a ratio of the previous data rates of the particular flow. Thus, the higher data rate the flow experiences in a certain period, the less chance for this flow to be selected at the next period, and eventually, more possibility for other flows to be selected. In this case fairness in of such traffic type is improved over different UEs. EXP-PF adopts a similar concept of fairness as in PF.

However, the system throughput is tuned exponentially and influenced by additional QoS parameters like queue size. This apparently seems to be in favor burst RT traffic that has a variable nature. Whereby, flows are selected based on their queue sizes weights to exponentially optimize throughput. This in turn provides high balance level between fairness that is obtained by the other part of the formula and throughput when compared with PF. With the similar notion as in EXP-PF, the EXP-Rule scheduler emphasizes more on maximiz- ing throughput on expense of fairness. EXP-Rule schedules flows belong to UE channel with high CQI. This typically maximizes the system throughput and spectrum efficiency. On the other hand, fairness in this case is derived only from the average weight of queue size. However, the relation between queue size and fairness does not guarantee that each flow has a sufficient service, whereby, flows with small queues may not be selected if other flows with huge queue are involved. The Log-Rule employs a logarithmic function on queue size. This allows small flows (VoIP or best effort) to have more chance to be scheduled while the service fairness of burst video traffic is maintained. Therefore, the relationship between fairness and throughput in this scheme is maintained robust and consistent regardless the offered load. 


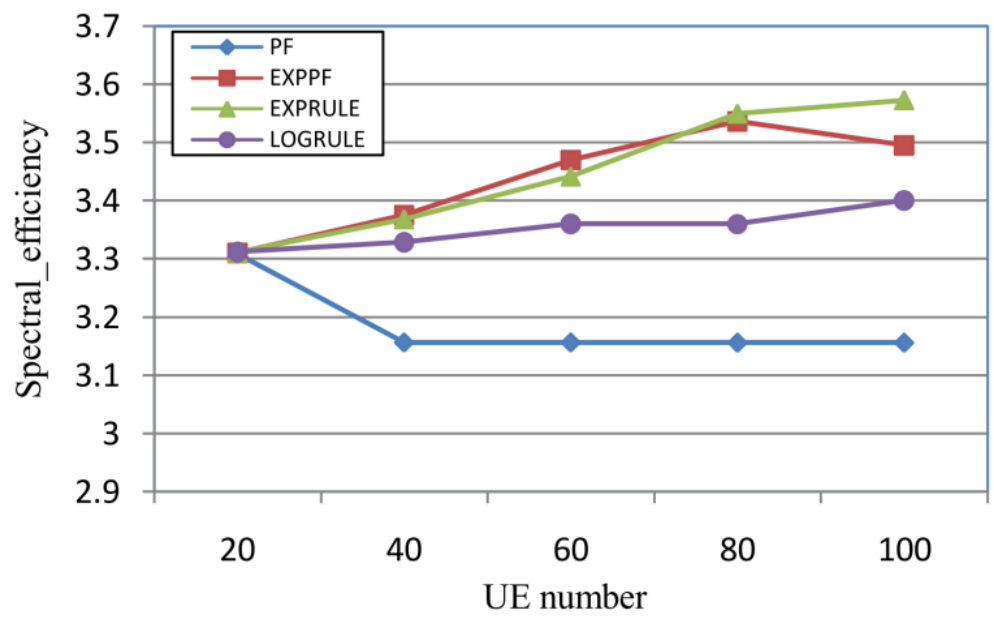

Fig. 6. Cell spectral efficiency

\section{Conclusion}

Based on the principle that a good scheduling scheme should increase system throughput while maintaining an accepted level of fairness to build the balance, four of the well-known scheduling schemes are studied and investigated. Assisted with simulation experiment scenario, the schedulers are evaluated in terms of fairness, system throughput, and the accumulative cell spectral efficiency. In short, it is inferred that Log-Rule most suitable scheduler when the cell operates in mixture of RT/NRT traffic as it succeeded to bring a good balance between throughput and fairness. From another side, EXP- PF and EXP-Rule are a good choice for RT traffic; hence EXP-PF is more fairness-friendly than EXP-Rule that takes throughput in first place. On the other hand, PF manages to keep NRT traffic QoS in a desired level, however; it suffers from wasting bandwidth resources, and its fairness is not accepted when channel is shared with RT traffic. Future directions of this work will extend to design an efficient scheduling scheme that is able to maintain the fairness to throughput balance in more complex scenario and meanwhile decreasing the delay on RT traffic to meet their QoS requirements.

\section{References}

[1] Ferdosian, N., Othman, M., Ali, B.M. and Lun, K.Y.: Greedyknapsack algorithm for optimal downlink resource allocation in LTE networks. Wireless Networks, pp. 1-14 (2015)

[2]Yuan-Ping,L.,Hu,B., Zhu, H. , Wei, Z., and Gao, W.:A delay priority scheduling algorithm for downlink real-time traffic in LTE networks. In Information Technology, Networking, Electronic and Automation Control Conference, IEEE, pp. 706-709. IEEE, 2016.

[3] Kwan, R. and Leung, C.: A survey of scheduling and interference mitigation in LTE. Journal of Electrical and Computer Engineering, vol. 2010, p. 1 (2010). 
[4] Tran, T.T., Shin, Y. and Shin, O.S.: Overview of enabling technologies for 3GPP LTE-advanced. EURASIP Journal on Wireless Communications and Networking, vol. 2012, p.1 (2012).

[5] Capozzi, F., Piro, G., Grieco, L.A., Boggia, G.and Camarda, P.: Downlink packet scheduling in LTE cellular networks: Key design issues and a survey. IEEE Communications Surveys and Tutorials, vol. 15, pp. 678-700 (2013).

[6] Cheng, H.T. and Zhuang, W.: An optimization framework for balancing throughput and fairness in wireless networks with QoS support. IEEE Transactions on Wireless Communications, vol. 7, pp. 584-593 (2008).

[7] Zabini, F., Bazzi, A. and Masini, B.M.: Throughput versus fairness tradeoff analysis. in 2013 IEEE International Conference on Communications (ICC), pp. 5131-5136 (2013).

[8] Afroz, F., Sandrasegaran, K. and Ghosal, P.: Performance analysis of PF, M-LWDF and EXP/PF packet scheduling algorithms in 3GPP LTE downlink. in Telecommunication Networks and Applications Conference (ATNAC), 2014 Australasian, pp. 87-92 (2014).

[9] Basukala, R., Ramli, H.M. and Sandrasegaran, K.: Performance analysis of EXP/PF and MLWDF in downlink 3GPP LTE system. in 2009 First Asian Himalayas International Conference on Internet, pp. 1-5 (2009).

[10] Alfayly, A., Mkwawa, I.H., Sun, L. and Ifeachor, E.: QoE-based performance evaluation of scheduling algorithms over LTE. in 2012 IEEE Globecom Workshops, pp. 1362-1366 (2012).

[11] Ramli, H.A.M., Basukala, R., Sandrasegaran, K. and Patachaianand, R.: Performance of well known packet scheduling algorithms in the downlink 3GPP LTE system. in Communications (MICC), 2009 IEEE 9th Malaysia International Conference on, pp. 815-820 (2009).

[12] AlQahtani, S.A. and Alhassany, M.: Comparing different LTE scheduling schemes. in 2013 9th International Wireless Communications and Mobile Computing Conference (IWCMC), pp. 264-269 (2013).

[13] Karipidis, E., Sidiropoulos, N.D. and Luo, Z.Q.: Quality of service and max-min fair transmit beamforming to multiple cochannel multicast groups. IEEE Transactions on Signal Processing, vol. 56, pp. 1268-1279 (2008).

[14] Huang, Y., Zheng, G., Bengtsson, M., Wong, K.K., Yang, L. and Ottersten, B.: Distributed multicell beamforming design approaching pareto boundary with max-min fairness. IEEE Transactions on Wireless Communications, vol. 11, pp. 2921-2933 (2012).

[15] Harks, T.: Utility proportional fair bandwidth allocation: An optimization oriented approach. in International Workshop on Quality of Service in Multiservice IP Networks, pp. 61-74 (2004).

[16] Parakh, S. and Jagannatham, A.K.: Game theory based dynamic bit-rate adaptation for H. 264 scalable video transmission in 4G wireless systems. in 2012 international conference on signal processing and communications (SPCOM), pp. 1-5 (2012).

[17] Khan, M.A. and Zaki, Y.: Dynamic spectrum trade and game-theory based network selection in lte virtualization using uniform auctioning. in International Conference on Wired/Wireless Internet Communications, pp. 39-55 (2011).

[18] Bechir, N., Mallouki, N., Mhatli, S., Ghanbarisabagh, M., Ammar, M. , and Hakimi, W.: Modeling and Performance Evaluation of Novel Scheduling Algorithm for Downlink LTE Cellular Network. Wireless Personal Communications 83, no. 3 (2015): 2303-2316.

[19] Qian, L., and Wen, C. C.: Smart Downlink Scheduling for Multimedia Streaming Over LTE Networks With Hard Handoff. IEEE Transactions on Circuits and Systems for Video Technology 25, no. 11 (2015).

[20] Ee, M.A., Wee, K., Han, Y.P., and Keong, K.P.: A performance analysis on packet scheduling schemes based on an exponential rule for real-time traffic in LTE." EURASIP Journal on Wireless Communications and Networking 2015, no. 1 (2015).

[21] Ahmed, A.A., Nordin, R., Ismail, M., and Abdullah, H.:An Efficient Scheduling Scheme for OFDMA Resource Blocks with Joint User Scheduling based on Earliest Deadline First with Carrier Aggregation (CA) in LTE-A System.” Wireless Personal Communications 88, no. 2 (2016). 
[22] Frank, K.,: Charging and rate control for elastic traffic, European transactions on Telecommunications, vol. 8, pp. 33-37, 1997.

[23] Hong, H.R, holtzman, J.M., Ku, K.D.: Scheduling of real/non-real time services: adaptive EXP/PF algorithm, in Vehicular Technology Conference, 2003. VTC 2003- Spring. The 57th IEEE Semiannual, 2003, pp. 462-466.

[24] Shakkottai, S. and Stolyar, A. L.:Scheduling for multiple flows sharing a time- varying channel: The exponential rule," Translations of the American Mathematical Society-Series 2, vol. 207, pp. 185-202, 2002.

[25] Sadiq, B., Madan, R., Sampath, A.: Downlink scheduling for multiclass traffic in LTE. EURASIP Journal on Wireless Communications and Networking, vol. 2009, p. 1, 2009.

[26] Sadiq, B., Jun, B., De, G.V.: Delay-optimal opportunistic scheduling and approximations: The log rule, IEEE/ACM Transactions on Networking (TON), vol. 19, pp. 405-418, 2011.

[27] Piro, G. , Grieco, L.A., Boggia, G., Cappozi, F., Camarda, P.: Simulating LTE cellular systems: an open-source framework. IEEE transactions on vehicular technology, vol. 60, pp. 498-513, 2011.

[28] Siping, L., Zhang, C., Zhou, Y., Zhang, Y.: Delay-Based Weighted Proportional Fair Algorithm for LTE Downlink Packet Scheduling. Wireless Personal Communications, vol. 82, pp. 1955-1965, 2015.

[29] Iwona, D., Jakubowski, M., Masiukiewicz, A., szeszko, M.: Fairness calculation on the base of the station media access time in Wi-Fi networks. in Information and Digital Technologies (IDT), 2016 International Conference on, 2016. 